\title{
Ophthalmic Manifestations In Thyroid Disease
}

\author{
Dr. M.Vijayaleela M.S ${ }^{1, D}$ D.D. BhimaSankarBabu M.S ${ }^{2}$ \\ ${ }^{1}$ Assosiate Professor OfOphthalmology Nizamabad \\ ${ }^{2}$ Associate Professor Of Ophthalmology SarojiniDevi Eye Hospital
}

\begin{abstract}
Out of 100 patients attending outpatient department of Ophthalmology, Sarojini Devi eye hospital Hyderabad, with history of Thyroid disease Thyroid eyedisease is clinically found in 26 patients(26\%).Majority of patients with thyroid eye disease are hyperthyroid $23 / 26(88.5)$ and $11.5 \%$ are hypothyroid(3/26).

Type Of Study:Retrospective study

Place Of Study:Sarojini Devi Eye Hospital

Aim Of The Study: To Study Various Manifestations In Thyroid Eye Disease.

Inclusion Criteria: All patient of age 50 years with systemic thyroid disease

Exclusion criteria:

1.Patient with other ocular pathologies

2.Patients with history of trauma.

Material And Methods: The study is done on 100 patients attending out patient department of ophthalmology, Sarojinidevi eye hospital, Hyderabadduring a periodof 2yearsi.e 2014-2016Apprpriate history is taken and through clinical examination of all system is done.Assesssment of visual acuity-both for distance using Snellen's chart and near using Snellen's near vision charts, colour vision using Ishihara's pseudo isocromatic charts, ocular movements, slit lamp examinations, measurement of proptosis, intra ocular pressure recording, fundus examination with direct and indirect ophthalmoscopy, visual field testing with Humphrey's automated visual field analyser.Laboratory investigations were done in all cases to know thyroid status and radiological investigations like ultrasound orbit and CT scan were done in relevant cases.
\end{abstract}

\section{Introduction}

Thyroid eye disease is also known as graves ophthalmopathy, dysthyroidophthalmopathy, thyroidassociated orbitopathy,thyroidorbitopathy, thyrotoxicexophthalmos.Orbital and related ocular changes are refered as thyroid orbitopathy or Graves' orbitopathy because it is an orbital rather than an ophthalmic process. Thyroid eye disease is the most common cause of unilateral or bilateral proptosis in adults and should always be a consideration in patients with unexplained diplopia,pain or optic nerve dysfunction.

TED was originally described as the part of the triad comprising Graves disease, which includes the orbital signs, hyperthyroidism, and pretibial myxedema.Grave's disease in an auto immune condition in which antibodies target the thyroid stimulating hormone receptor(TSH-R) displayed on thyrocytes, inducing the production of excess thyroid hormones. Though typically associated with graves hyperthyroidism TED may also occur with Hashimoto thyroiditis or in the absence of thyroid disease. Subclinical thyroid abnormalities may be noted in some patients with typical orbitopathy.

The majority of patients have clinical or laboratory evidence of thyroid disease but the orbital process can occur even in the absence of detectable thyroid abnormality. Thyroid orbitopathy is overwhelmingly associated with and usually occurs close to or within 18 months of hyperthyroidism; there is a rough correlation between the nature of hyperthyroidism, it's management, and the orbitopathy.

Median age at the time of diagnosis of Graves' ophthalmopathy was 43 years; the minimum and maximum ages were 8 and 88 years,respectively. Among 108 patients with hyperthyroidism, ophthalmopathy was diagnosed in the six-month interval preceeding the diagnosis of thyroid dysfunction in 20 patients(18.5\%); opthalmopathy-was concurrent with the diagnosis of hyperthyroidism in 22 patients(20.3\%); and ophthalmopathy developed in the six-month interval after thyroid-diagnosis in 24 patients(22.2\%). Ophthalmopathy was diagniosed more than six months before the diagnosis of hyperthyroidism in only four additional patients $(3.7 \%)$, where as ocular changes developed six months or more after thyroid disease in the remaining 38 patients $(35.2 \%)$.

Thyroid eye disease(TED) causes visual disability, discomfort, and facial disfigurement. These symptoms and signs are caused by immune-mediated, inflammatory events that may result in irreversible tissue alterations.Considerable controversy exists as to whether treatment is better than observation during the active phase of disease. Management should consist of a coordinated, multidisciplinary, medical surgical approach based on staging of the disease and knowledge of its effect on the orbital and ocular structures.But even with 
appropriate interventions at each stage of the disorder,TED patients often suffer a dramatic reduction in their quality of life.

Broadly speaking, management is directed toward abating or controlling the active phase of the disease, prevention of ocular and psychophysical damage, regressing ocular motor abnormalities, and improving the cosmetic disfigurement.

\section{Epidemiology}

- In a study of patients Olmsted Country, the age-adjusted incidence of thyroid eye disease(TED) for women was 16 cases, and for men it was 2.9 cases per 100,000 population per year.

- Thyroid Eye Disease patients with thyroid dysfunction appear to have more severe ophthalmopathy than those who are clinically euthyroid.

\section{Race}

- Tellez et al. reported a 6.4 times risk for development oforbotopathy in Europeans compared to Asians.

- They also found that the prevelence of Thyroid Associated Orbitopathy in patients with hyperthyroidism was singificantly higher in Europeans(42\%) compared to Asians(7.7\%).

Sex

- Thyroid eye disease affects women in approximately 6 times more frequently than men(86\% versus $14 \%$ of cases, respectively)

Age

- There was a bimodal peak incidence in the fifth and seventh decades in both men and women. While Thyroid Eye Disease is more common in younger women, the disorder is more severe in men and patients over the age of 50-60 years.

- The median age at the time of diagnosis of TED was 43 years(range, 8-88 years).

\section{Smoking}

- Smokers are upto 5 times more likely than nonsmokers to develop Thyroid Eye Disease.

- Cigarette smoking is the strongest modifiable risk factor for developing thyroid Associated Orbitopathy.

- The risk and severity of ophthalmopathy in patients with graves disease may be increases by several other factors including tobacco use,genetics, type of treatment for hyperthyroidism, TSH receptor levels, advanced age and stress.

- History Of Thyroid Eye DiseaseSaint Yves was the first ophthalmic surgeon who made reference to thyroid ophthalmopathy in 1773.

- Caleb Hillier Parry gave a detailed clinical description of 13 cases of goitre associated with tachycardia, in one of which, protrusion of eyes occurred for the first time, the condition has sometimes been called Parry's disease.

- Robert James Gravesdescription of enlargement of the thyroid, palpitations and protrusion of the eyes is so complete and detailed that the connation Grave's Disease has been accepted throughout the world.

- SArl Adolph Von Basedowdescribed exophthalmos as due to hypersecretion of the orbital tissue caused by disordered circulation, discussing the three cardinal symptoms of exophthalmos, goitre and tachycardia.The disease is frequently called Basedow's disease in German literature.

- Classification Of Thyroid Eye Diseasein1969, Warner proposed the "No Specs" classification for signs of Graves' ophthalmopathy. In 1981, Van Dyke refined the class 2 No Specs soft tissue findings with the mnemonic Relief

- --no specs" and "relief" categorization of graves' disease

- class signs

- $\quad$ 0. No signs nor symptoms

- 1. Only signs are upper eyelid retraction, lid lag, stare

- 2. Soft tissue signs and symptoms:

- Resistant to retropulsion

- Edema of conjuctiva and caruncle

- Lacrimal gland enlargement

- Injection over horizontal rectus muscle insertions

- Edema of the eyelids

- 3.Proptosis

- 4.Extraocular muscle involvement

- 5.Corneal involvement secondary to exposure 
- 6.Sight loss secondary to optic nerve compression

\title{
II. Clinical Features
}

Common and non specific symptoms include tearing, irritation, giddiness, arching, and photophobia.

Early signs include conjuctival injection, periorbital puffiness, abnormal tear break-up time, superficial punctate keratitis, and elevation of intra ocular pressure.Ophthalmic findings are generally bilateral, but may present unilaterally or asymmetrically. The presence of pre-existing autoimmune thyroid disease increases suspicion for thyroid eye disease, but isolated eye findings may represent the presenting manifestations of thyroid disorders. The classic presentation in the setting of acute Graves' disease involves thyrotoxicosis,goiter and bilateral exophthalmos

The clinical signs are characteristic and include 1 or more of the following:

- Proptosis

- Restrictive extraocular myopathy

- Eyelid retraction

- Lid lag

- Corneal exposure

- Compressive optic neuropathy

Excessive tearing,foreign body sensation,bulging of the eyes, swelling of the eyelids,retro-orbital pain, blurred vision, inability to focus, double vision characterize early, active thyroid Eye disease.

Thyroid Eye Disease dysmotility often presents as intermittent diplopia that is initially more symptomatic in the morning due to edema thatresults from sleeping in a supine position. In 5-10\% of thyroid eye disease patients, visual loss occurs due to corneal decompensation or optic nerve compression. $25 \%$ to $50 \%$ of patients with Graves' hyperthyroidism will have some features of ophthalmopathy.

\section{Thyroid Eye Signs}

1. Dalrymple's-upper lid retraction

2. Von Graefe'supperlid lag on downwarg gaze

3. Boston's jerky irregular movement of upper lid on downward gaze

4. Enroth's puffy swelling of lids

5. Giffords difficulty in everything upperlid.

6. Gellinek sign increased pigmentation of lids

7. Kocher's sign increased lid retraction with visual fixation

8. Rosenbach's tremor of closed lids

9. Stellwag's infrequent blinking

10. Mobius weakness of convergence

11. Ballet's sign palsy of one or more extraocular muscles

12. Suker'ssignweakness of fixation on lateral gaze

13. Cowen's sign jerky papillary constriction to consensual light

14. Knies sign unequal dilation of pupil

15. Lowy's sign dilation of pupil with weak adrenaline

16. Joffroy's absence forhead wrinkles on upward gaze

17. Saintons's delayed forhead wrinkling after upward gaze.

18. Payne Trouseau sign globe luxation

19. Vigoroux sign lid swelling

20. Pochin sign diminished blinking

\author{
III. Medical Treatment \\ Treatment of Thyroid Disease and Prevention of Thyroid Orbitopathy \\ 1. Oral antithyroid drugs \\ Advantages- \\ i. Control of hyperthyroidism \\ ii. Improvement in the orbitopathy
}

\section{Radioactive iodine}

In some studies, thyroid eye disease has been demonstrated to worsen after RAI treatment, presumably because of the release of TSH-R antigens which incite an enhanced immune response.In addition,Hypothyroidism occurring after RAI treatment may exacerbate thyroid eye disease via stimulation of TSH-R. 
Hyperthyroid patients with severe, active thyroid eye disease; those with elevated T3,levels; and smokers appear to be at greatest risk for exacerbation of eye disease after RAI treatment.33

Disadvantages -progression of subclinical and clinical orbitopathy which is prevented by combining with prednisolone $25-50 \mathrm{mg} / \mathrm{day}$ for 3 months.

\section{Thyroidectomy}

Advantages

1.Eliminates reactive $\mathrm{T}$ lymphocytes in the thyroid.

2. Improves orbitopathy

Disadvantages

- Surgical complication is reaction of parathyroids

Mild disease

\section{Management Of Thyroid Eye Disease}

- Observation

- Patient education/lifestyle changes

- Smoking cessation

- Salt restriction

- Elevation of head of bed

- Wearing sunglasses

- Ocular surface lubrication

\begin{tabular}{|l|l|}
\hline Signs/Symptoms & Management Options \\
\hline Peri-orbital oedema & Elevation head of the bed, antidiuretics \\
\hline Dryness, foreign body sensation & Artificial eye drops and ointment \\
\hline Lagophthalmos & Nocturnal eye taping,eyes shield \\
\hline Eyelid retraction & $\begin{array}{l}\text { Topical Guanethidine drops or beta } \\
\text { blockers eye drops }\end{array}$ \\
\hline Diplopia & Prisms \\
\hline Photophobia & Sunglasses \\
\hline
\end{tabular}

\section{Moderate disease}

- Topical cyclosporine

- Eye lid taping at night

- Moisture goggles/chambers

- Prism glasses or selective ocular patching

- Moderate-dose oral steroid therapy

\section{Surgical Management}

For surgical rehabilitation of the patient with stable thyroid eye disease, surgical repair is recommended in the following standard, sequential stages:

1. Orbital decompression surgery

2. Eye muscle surgery

3. Eyelid repositioning

4. Blepharoplasty(which is sometimes combined with eyelid repositioning)

The preoperative CT scan details the relative contributions of extraocular muscle enlargement and fat expansion to the proptosis.Patients younger than 40 years demonstrate enlargement of the orbital fat compartment, whereas those older than 40 typically show more significant extraocular muscle enlargement. This difference determine the effectiveness of bone versus fat decompression surgery.

\section{Orbital Decompression}

Orbital decompression is a surgical procedure used to improve the volume-to-space discrepancy.The goal of orbital decompression is to allow the enlarged muscles and orbital fat to expand into periorbital spaces. This expansion relatives on the optic nerve and its blood supply and reduces proptosis.

\section{Technical options in decompression}

A. Floor and medial wall

1. Anterior

Medial skin 


\section{Caruncular}

2. sinus approachOguraTransnasal endoscopic

B. Lateral wall+_floor+_roof+_augmentation or bony advancement

1. Swinging eyelid approach

2. Burke-Kronlein+medical approach

3. Burke-Kronlein+Ogura or transnasal endoscopic

4. Coronal approach

C. Soft tissue

1.Orbital fat excision

2.Blepharoplasty

External

Internal

There are only two instances in which relatively emergent surgery is necessary,And decompression surgery involves the removal of 1,2,3, or(seldom) 4 orbital walls enlarge the orbit and reduce proptosis and/or optic nerve compression.

\section{Concurant orbital fat exicision is used to augment the impact of surgery.}

- Surgical approaches include

- Transeyelid

- Transcaruncular

- Transantral

- Transnasal endoscopic

- Andcoronal

\section{Results}

Age at Presentation among Thyroid eye disease patients

\begin{tabular}{|l|l|}
\hline Mean age of Presentation & Percentage \\
\hline $0-9$ & $0 \%$ \\
\hline $10-19$ & $3.84 \%$ \\
\hline $20-29$ & $23.07 \%$ \\
\hline $30-39$ & $19.23 \%$ \\
\hline $40-49$ & 53.84 \\
\hline
\end{tabular}

Most common age group at presentation is $40-49$ years followed by $30-39$ years

Table-2Sex distribution among thyroid eye disease patients

\begin{tabular}{|l|l|l|}
\hline Sex & No.Of Cases & Percentage \\
\hline Females & 19 & $73 \%$ \\
\hline Males & 7 & $26.9 \%$ \\
\hline
\end{tabular}

$73 \%$ Thyroid Eye Disease patients are females and $26.9 \%$ are males.

Table-3Smokers Vs Non Smokers

\begin{tabular}{|l|l|}
\hline Smoking Status & Percentage \\
\hline Smokers & $34.61 \%$ \\
\hline Non-smokers & $65.39 \%$ \\
\hline
\end{tabular}

34.61\% of Thyroid Eye Disease patients are smokers and $65.39 \%$ are non smokers.

Table-4Thyroid status

\begin{tabular}{|l|l|l|}
\hline Thyroid Status & No.Of Cases & Percentage \\
\hline Hyperthyoidism & 23 & $88 \%$ \\
\hline Hypothyroidism & 3 & $11.5 \%$ \\
\hline
\end{tabular}

$88 \%$ of the thyroid disease patients are hyperthyroid and $11.5 \%$ patients are hypothyroid.

Table-5Laterality

\begin{tabular}{|l|l|l|}
\hline Laterality & No.Of Cases & Percentage \\
\hline Bilateral & 24 & $92.3 \%$ \\
\hline Unilateral & 2 & $7.69 \%$ \\
\hline
\end{tabular}


$92.31 \%$ of thyroid eye patients have bilateral involvement when as $7.69 \%$ have unilateral involvement.

Table-6Frequency of occurance of various manifestations in Thyroid eye disease

\begin{tabular}{|l|l|}
\hline Manifestation & Percentage \\
\hline Eyelid retraction & $88.46 \%$ \\
\hline Proptosis & $57.69 \%$ \\
\hline Restrictive myopathy & $38.46 \%$ \\
\hline Exposure Keratitis & $3.84 \%$ \\
\hline Optic neuropathy & Nil \\
\hline
\end{tabular}

Eyelid retraction is the most common manifestation occurring in $88.46 \%$ of Thyroid eye disease patients followed by proptosis in $57.69 \%$, Restrictive myopathy in $38.46 \%$, Exposure Keratitis in $3.84 \%$.

\section{Discussion}

The focus of the present study was to know the ophthalmic manifestations in thyroid disorders.Out of 100 patients attending outpatient department of Ophthalmology thyroid eye disease is clinically found in 26 patients(26\%).Majority of patients with thyroid eye disease are hyperthyroid $23 / 26(88.5)$ and $11.5 \%$ are hypothyroid(3/26).

\section{Conclusions}

A total of 100 cases of thyroid disorder were examined and evaluated during the study period.

- Majority of Thyroid eye disease patients are hyperthyroid(88.5\%).

- Few Thyroid eye disease patients are hypothyroid(11.5\%).

- Thyroid eye disease is common in females when compared with males.

- Mean age of presentation is 40 years.

- It is mostly bilateral.

- Smoking is associated with increased risk and severity of disease, followed by proptosis, then comes soft tissue signs.

- $\quad$ Sight threatening complication occurred in 5\% of Thyroid eye disease patients.

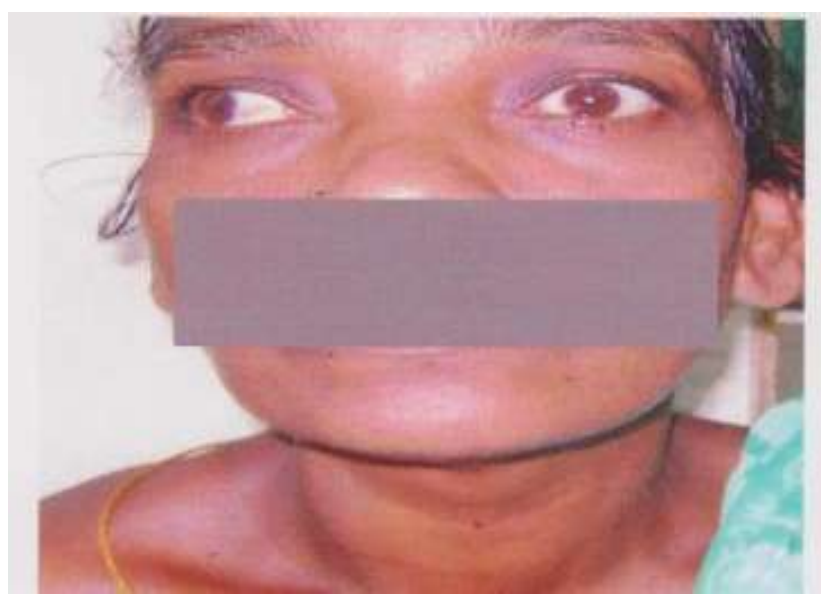

Thyroid eye disease with Restrictive myopathy

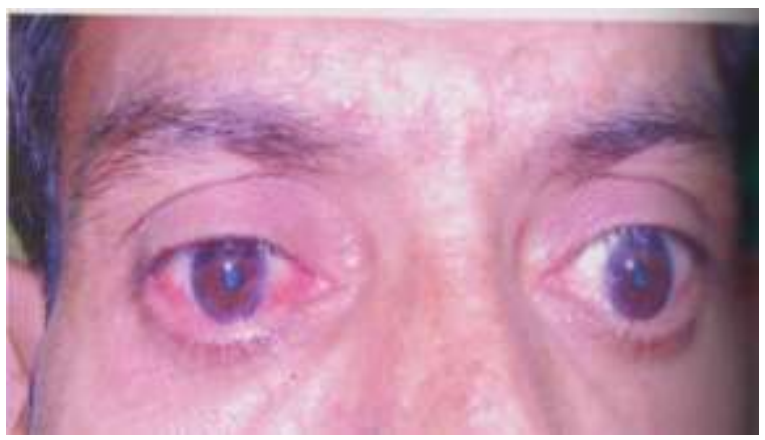

Right eye showing congestion over the insertion of horizontal recti 


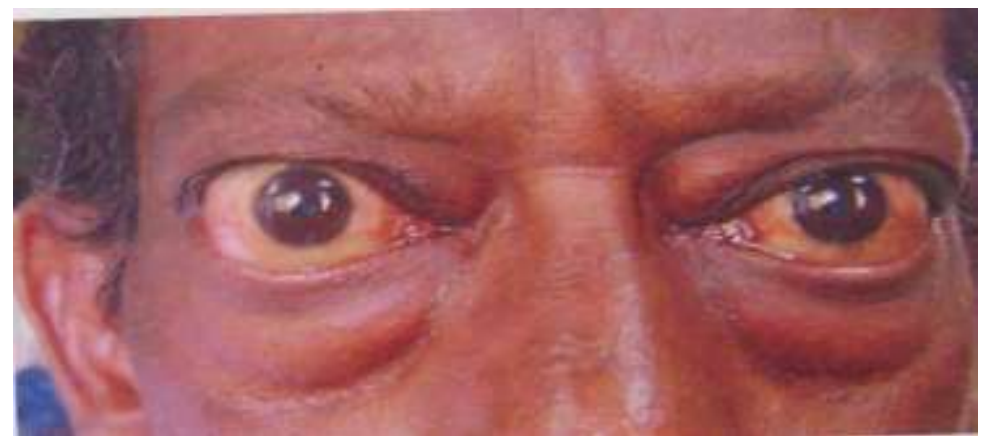

Both eye puffy swelling of eye lids

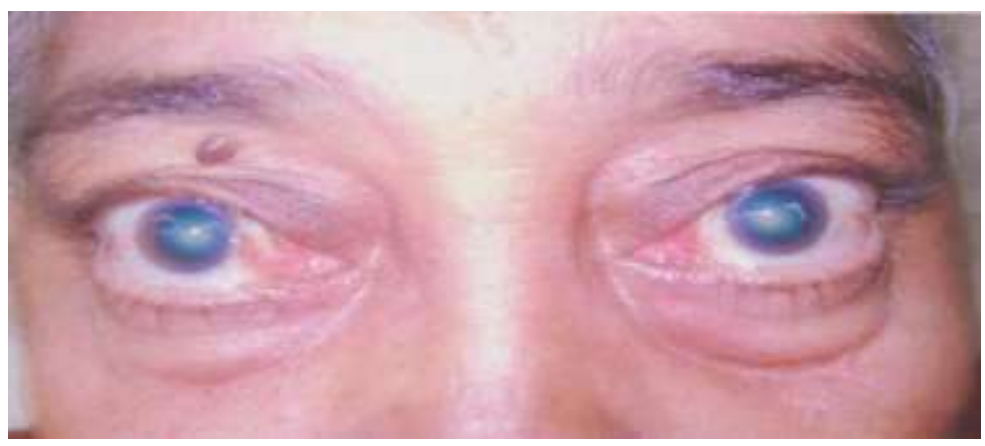

Both Eye Eyelid retraction and puffyness of eye lids

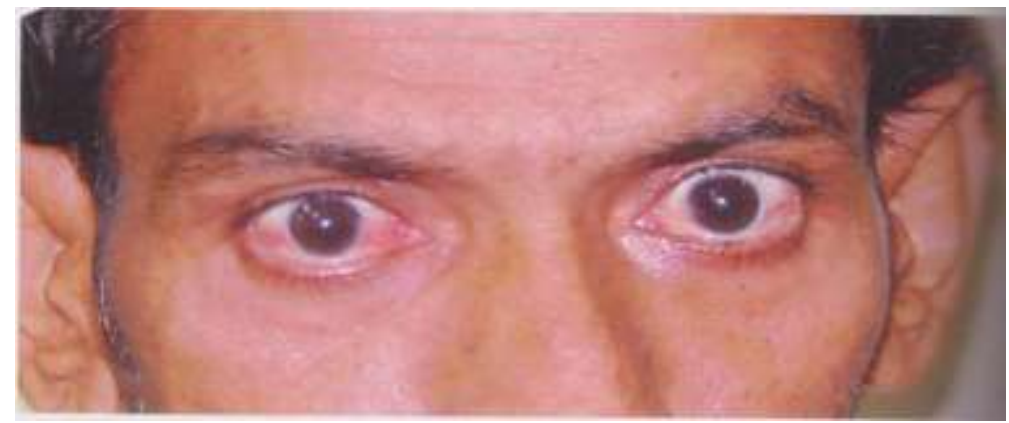

Both eyes Eyelid retraction and proptosis

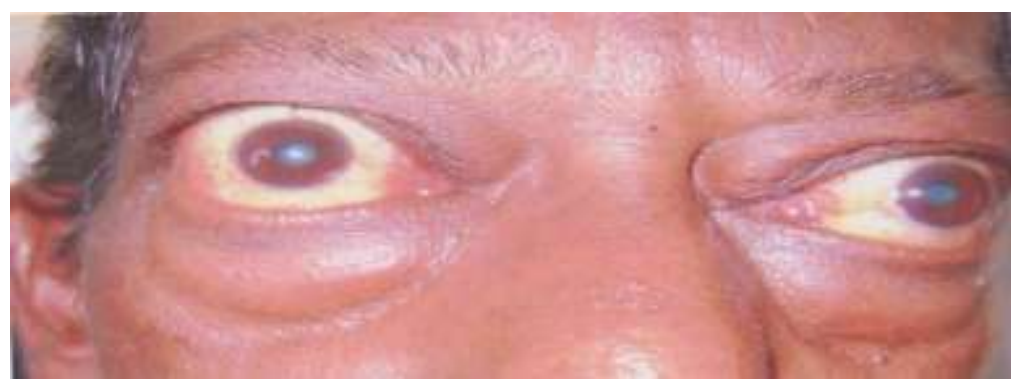

Both eyes eyelid retraction and proptosis

\section{Bibliography}

[1]. Prabhakar BS, Bahn RS, Smith TJ. Current perspective on the pathogenesis of Graves' disease and ophthalmology. Endocrinology Review 2003;24:802-35

[2]. Am J Ophthalmol. 1996 Apr; 121(4) : 426-34. Chronolgy of Graves-ophthalmopathy in an incidence cohort.

[3]. Indian Journal of ophthalmology Vol.60 No.2 March 2012 Thyroid associatalorbotopathy.

[4]. Tellez M, Cooper J, Edmonds C. Graves ophthalmopathy in relation to cigarette smoking and ethnic origin. ClinEndocrinol(Oxf)1992;36:291-4

[5]. Kendler DL, Lippa J, Rootman J. The initial clinical characteristics of Grave's orbitopathy vary with age and sex.ArchOphthalmol. 1993; 111(2):197-201. 